\title{
antibiotics
}

ISSN 2079-6382

www.mdpi.com/journal/antibiotics

Article

\section{Induction of Antimicrobial Resistance in Escherichia coli and Non-Typhoidal Salmonella Strains after Adaptation to Disinfectant Commonly Used on Farms in Vietnam}

\section{Nguyen T. Nhung ${ }^{1, *}$, Cao T. Thuy ${ }^{1}$, Nguyen V. Trung ${ }^{1,2}$, James Campbell ${ }^{1,3}$, Stephen Baker ${ }^{1,3}$,} Guy Thwaites ${ }^{1,3}$, Ngo T. Hoa ${ }^{1,3}$ and Juan Carrique-Mas ${ }^{1,3}$

1 The Hospital for Tropical Diseases, Wellcome Trust Major Overseas Programme, Oxford University Clinical Research Unit, Ho Chi Minh City, Vietnam;

E-Mails: thuyct@oucru.org (C.T.T.); trungnv@oucru.org (N.V.T.); jcampbell@oucru.org (J.C.); sbaker@oucru.org (S.B.); gthwaites@oucru.org (G.T.); hoant@oucru.org (N.T.H.); jcarrique-mas@oucru.org (J.C.-M.)

2 Department of Medical Microbiology, Academic Medical Center, University of Amsterdam, Amsterdam $1105 \mathrm{AZ}$, the Netherlands

3 Centre for Tropical Medicine, Nuffield Department of Clinical Medicine, Oxford University, Old Road Campus, Roosevelt Drive, Oxford, OX3 7BN, UK

* Author to whom correspondence should be addressed; E-Mail: nhungnt@oucru.org; Tel.: +84-83-923-7954; Fax: +84-39-238-904.

Academic Editor: Martin J. Woodward

Received: 16 June 2015 / Accepted: 27 October 2015 / Published: 30 October 2015

\begin{abstract}
In Vietnam, commercial disinfectants containing quaternary ammonium compounds (QACs) are commonly used in pig and poultry farms to maintain hygiene during production. We hypothesized that sustained exposure to sub-bactericidal concentrations of QAC-based disinfectants may result in increased levels of antimicrobial resistance (AMR) among Enterobacteriacea due to the increase of efflux pump expression. To test this hypothesis we exposed six antimicrobial-susceptible Escherichia coli (E. coli) and six antimicrobial-susceptible non-typhoidal Salmonella (NTS) isolates to increasing concentrations of a commonly used commercial disinfectant containing a mix of benzalkonium chloride and glutaraldehyde. Over the 12-day experiment, strains exhibited a significant change in their minimum inhibitory concentration (MIC) of the disinfectant product (mean increase of $31 \%(\mathrm{SD} \pm 40))(p=0.02$, paired Wilcoxon test). Increases in
\end{abstract}


MIC for the disinfectant product were strongly correlated with increases in MIC (or decreases in inhibition zone) for all antimicrobials (Pearson's correlation coefficient $0.71-0.83$, all $p<0.01$ ). The greatest increases in MIC (or decreases in inhibition zone) were observed for ampicillin, tetracycline, ciprofloxacin, and chloramphenicol, and the smallest for gentamicin, trimethoprim/sulphamethoxazole. The treatment of 155 representative E. coli isolates from farmed and wild animals in the Mekong Delta (Vietnam) with phenyl-arginine beta-naphthylamide $(\mathrm{PA} \beta \mathrm{N})$, a generic efflux pump inhibitor, resulted in reductions in the prevalence of AMR ranging from $0.7 \%$ to $3.3 \%$ in these organisms, indicating a small contribution of efflux pumps on the observed prevalence of AMR on farms. These results suggest that the mass usage of commercial disinfectants, many of which contain QACs, is potentially a contributing factor on the generation and maintenance of AMR in animal production in Vietnam.

Keywords: disinfectant; antimicrobials; adaptation; efflux pumps

\section{Introduction}

Antimicrobial resistance (AMR) is a major global health threat recognized by the research community as well as by international agencies concerned with human and veterinary medicine [1,2]. The intensity of antimicrobial use is firmly established as one of the key drivers for the development of AMR both in human and veterinary medicine $[3,4]$.

Disinfectants are substances applied to kill microorganisms on inanimate objects. In contrast to antimicrobials, which act on specific bacterial targets, disinfectants have multi-factorial mechanisms of action [5]. The use of disinfectants at the end of the food animal production cycle (i.e., terminal cleaning and disinfection) is a crucial procedure for limiting the transmission of infections between animals sequentially housed in the same buildings, therefore contributing to overall flock/herd health and productivity [6]. There has been mounting evidence that exposure to certain disinfectant agents may induce reduced susceptibility and cross-resistance with antimicrobial agents [7-9].

Quaternary ammonium compound (QAC)-based disinfectants are some of the most commonly used in industry and in animal production because of their stability and low cytotoxicity, as well as being non-corrosive on farm equipment [10]. The potential cross-resistance between QACs and antimicrobial agents has been a particular concern over recent years $[11,12]$. The increased expression of multidrug efflux pumps has been proposed to be responsible for decreased susceptibility to both QACs and antimicrobials [13].

Recent studies have shown a high prevalence of AMR among Enterobacteriaceae from Vietnamese livestock and poultry farms [14-16]. We hypothesized that disinfectants commonly used on farms may be one factor contributing to AMR due to unintentional dilution after application on occupied buildings and a large proportion of commercial products include QACs in their formulation. To test this hypothesis we exposed 12 pan-susceptible Enterobacteriaceae strains to suboptimal levels of a 
widely used commercial product containing benzalkonium chloride and glutaraldehyde (Product A) and measured changes in their tolerance to the product as well as changes in susceptibility to a range of important antimicrobial agents. In order to investigate whether generic efflux pump expression was responsible for the observed changes, we treated strains with a generic efflux pump inhibitor and measured the changes in AMR before and after treatment.

\section{Results}

\subsection{Adaptation of Strains to Product A}

We investigated 12 pan-susceptible Enterobacteriacea strains (six E. coli and six non-typhoidal Salmonella (NTS) strains) before and after in vitro exposure to commercial Product A, which contained $150 \mathrm{mg} / \mathrm{mL}$ of benzalkonium chloride and $150 \mathrm{mg} / \mathrm{mL}$ of glutaraldehyde. After the 12-day exposure period, six of 12 strains increased their MIC for Product A (i.e., $\mu \mathrm{g} / \mathrm{mL}$ of both active components).

An increase was observed for four of six field NTS strains and for two of six field E. coli strains. One E. coli strain reduced its MIC after the adaptation period (Table 1).

After the adaptation period, the overall mean MIC of Product A (12 strains, three experimental replicates per strain, i.e., 36 observations) increased from $21 \mu \mathrm{g} / \mathrm{mL}(\mathrm{SD} \pm 6)$ to $27 \mu \mathrm{g} / \mathrm{mL}$ ( $\mathrm{SD} \pm 12$ ) (i.e., a $31 \%$ ( $\mathrm{SD} \pm 40)$ increase) ( $p=0.02$, paired Wilcoxon test) (Table 1). The observed variability in MIC between experimental replicates was minimal, with a median coefficient of variation of $0 \%$ (75\% inter-quartile range (IQR) $0-11.3 \%$ ) and $0 \%$ (75\% IQR 0-14.7\%) for MIC readings before and after the adaptation experiment, respectively.

Strains did not significantly change their MIC after culture in disinfectant-free media (Mueller Hinton $(\mathrm{MH})$ broth at $\left.37{ }^{\circ} \mathrm{C}\right), 22 \mu \mathrm{g} / \mathrm{mL}(\mathrm{SD} \pm 5)$ compared with a baseline average $21 \mu \mathrm{g} / \mathrm{mL}$ $(\mathrm{SD} \pm 6)$, (i.e., $\mathrm{a}+5 \%(\mathrm{SD} \pm 19)$ of change) $(p=0.19$, Wilcoxon test $)$.

\subsection{Relationship between Changes in MIC for Product A and Changes in Antimicrobial Susceptibility}

We observed a strong positive correlation between increased MICs for Product A and increased MICs for gentamicin, chloramphenicol, ciprofloxacin, trimethoprim/sulphamethoxazole, and tetracycline (Pearson's correlation coefficients (r) ranging from 0.71 to 0.82 ; all $p \leq 0.01$ ). Increases in MIC for Product A were also strongly correlated with reductions in zone diameter in the ampicillin disc diffusion test $(r=0.83 ; p<0.001)$ (Figure 1). 
Table 1. Minimum inhibitory concentrations (MIC) against studied strains for Product A. Columns 3, 4, and 6 show MICs ( $\mu \mathrm{g} / \mathrm{mL}$ ) pre-exposure, post-exposure, and after treatment with efflux pump inhibitor (PA $\beta \mathrm{N})$. Column 5 shows changed MIC levels (\%) after exposure to Product A compared with pre-exposure levels. Column 7 shows the average decreased MIC (\%) after treatment with efflux pump inhibitor compared with post-exposure levels.

\begin{tabular}{|c|c|c|c|c|c|c|}
\hline \multirow[b]{2}{*}{ Strain ID } & \multirow[b]{2}{*}{ Species } & \multicolumn{2}{|c|}{ Average MIC $(\mu \mathrm{g} / \mathrm{mL})$} & \multirow{2}{*}{$\begin{array}{c}\text { Average Change in } \\
\text { MIC after } \\
\text { Exposure }\end{array}$} & \multirow{2}{*}{$\begin{array}{c}\text { Average MIC }(\mu \mathrm{g} / \mathrm{mL}) \\
\text { after Treatment with } \\
\text { PA } \beta N\end{array}$} & \multirow{2}{*}{$\begin{array}{c}\text { Average Change in } \\
\text { MIC after Treatment } \\
\text { with PA } \beta \text { N }\end{array}$} \\
\hline & & Pre-exposure & Post-exposure & & & \\
\hline S01 & $S$. Derby & 21 & 21 & $0 \%$ & 15 & $-29 \%$ \\
\hline S02 & S. Ohio & 24 & 33 & $+38 \%$ & 24 & $-27 \%$ \\
\hline S03 & $S$. Weltevreden & 24 & 33 & $+38 \%$ & 27 & $-18 \%$ \\
\hline S04 & $S$. Weltevreden & 30 & 60 & $+100 \%$ & 36 & $-40 \%$ \\
\hline S05 & $S$. Newport & 24 & 36 & $+50 \%$ & 30 & $-17 \%$ \\
\hline $\mathrm{SC}$ & $\begin{array}{c}S . \text { Typhimurium } \\
14028^{*}\end{array}$ & 24 & 24 & $0 \%$ & 24 & $0 \%$ \\
\hline E01 & E. coli & 15 & 15 & $0 \%$ & 15 & $0 \%$ \\
\hline E02 & E. coli & 27 & 24 & $-11 \%$ & 15 & $-38 \%$ \\
\hline E03 & E. coli & 12 & 12 & $0 \%$ & 12 & $0 \%$ \\
\hline E04 & E. coli & 12 & 24 & $+100 \%$ & 18 & $-25 \%$ \\
\hline E05 & E. coli & 27 & 42 & $+56 \%$ & 33 & $-21 \%$ \\
\hline $\mathrm{EC}$ & E. coli $25922 *$ & 12 & 12 & $0 \%$ & 12 & $0 \%$ \\
\hline \multicolumn{2}{|c|}{ All isolates (mean $\pm \mathrm{SD})$} & $21(\mathrm{SD} \pm 6)$ & $27(\mathrm{SD} \pm 12)$ & $+31 \%(\mathrm{SD} \pm 40 \%)$ & $21(\mathrm{SD} \pm 9)$ & $-18 \%(\mathrm{SD} \pm 15 \%)$ \\
\hline
\end{tabular}

SD: Standard deviation; * Laboratory reference strains. 

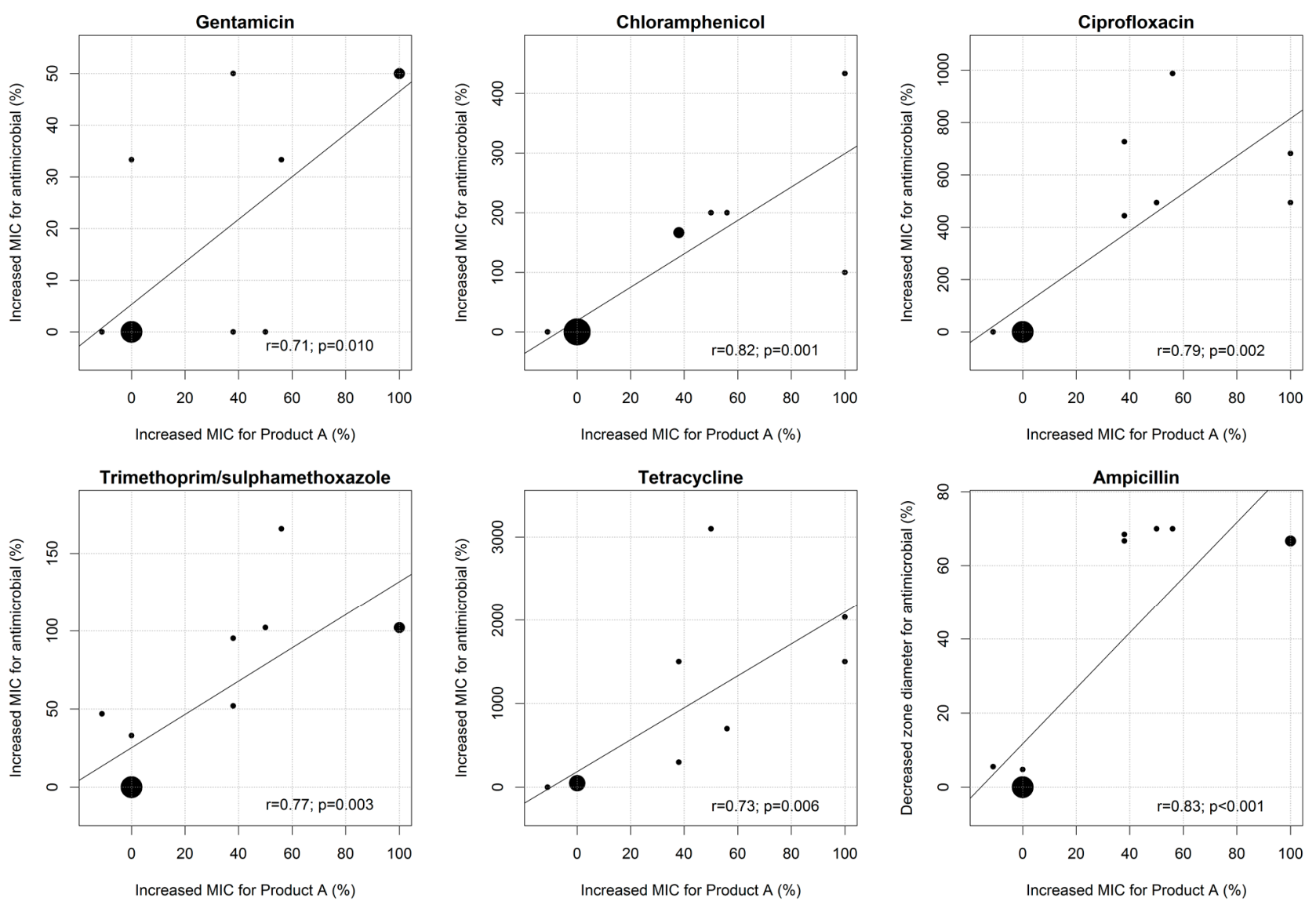

Figure 1. Correlation between changes in MIC for Product A and changes in MIC for gentamicin, chloramphenicol, ciprofloxacin, trimethoprim/sulphamethoxazole, and tetracycline. The size of each dot is proportional to the number of strains.

\subsection{Changes in Antimicrobial Susceptibility before and after Exposure to Product A}

After exposure to Product A, there were overall MIC increases for all antimicrobials tested (Table 2). The largest changes in MIC were observed for tetracycline (mean change $+776 \%$, SD \pm 1027 ), followed by ciprofloxacin $(+316 \%, \mathrm{SD} \pm 363)$, chloramphenicol $(+106 \%$, SD \pm 135$)$, trimethoprim/ sulphamethoxazole $(+58 \%, \mathrm{SD} \pm 55)$, and gentamicin $(+18 \%, \mathrm{SD} \pm 23)$. The average inhibition zone of ampicillin decreased from $18.6 \mathrm{~mm}(\mathrm{SD} \pm 1.5)$ to $12.0 \mathrm{~mm}(\mathrm{SD} \pm 6.4)$. Qualitative modifications in the AMR profile were demonstrated for tetracycline (three NTS and two E. coli isolates developed full resistance and one NTS strain exhibited intermediate resistance), chloramphenicol (four NTS and two E. coli isolates exhibited intermediate resistance), and ampicillin (the six strains that showed an increased MIC for Product A also developed ampicillin resistance). After the adaptation experiment, all strains remained susceptible to ciprofloxacin, gentamicin, and trimethoprim/sulphamethoxazole (Table 2 and Figure 2). 
Table 2. MIC against studied strains for gentamicin, chloramphenicol, ciprofloxacin, trimethoprim/sulphamethoxazole, and tetracycline; zone inhibition diameter for ampicillin before exposure to Product A, after exposure to Product A, and after treatment with efflux pump inhibitor $(\mathrm{PA} \beta \mathrm{N})$. The $p$-values were obtained using paired Wilcoxon tests for the comparisons: ${ }^{\dagger}$ before and after exposure to Product $\mathrm{A} ;{ }^{\S}$ after exposure to Product A and after treatment with efflux pump inhibitor.

\begin{tabular}{|c|c|c|c|c|c|c|c|}
\hline \multirow{2}{*}{ Antimicrobial } & \multicolumn{2}{|c|}{$\operatorname{Mean}( \pm$ SD) $(\mu \mathrm{g} / \mathrm{mL})$} & \multirow{2}{*}{$\begin{array}{c}\text { Mean Change } \\
(\%)( \pm \text { SD })\end{array}$} & \multirow{2}{*}{$p$-Value ${ }^{\dagger}$} & \multirow{2}{*}{$\begin{array}{c}\text { Mean }( \pm \text { SD }) \\
(\mu \mathrm{g} / \mathrm{mL}) \\
\text { After Treatment } \\
\text { with PA } \beta \mathbf{N} \\
\end{array}$} & \multirow{2}{*}{$\begin{array}{c}\text { Mean Change } \\
(\%)( \pm \text { SD })\end{array}$} & \multirow{2}{*}{$p$-Value ${ }^{\S}$} \\
\hline & Pre-exposure & Post-exposure & & & & & \\
\hline Gentamicin * & $1.12( \pm 0.29)$ & $1.29( \pm 0.26)$ & $+18( \pm 23)$ & 0.053 & $1.21( \pm 0.26)$ & $-6( \pm 13)$ & 0.346 \\
\hline Chloramphenicol * & $6.50( \pm 1.51)$ & $13.67( \pm 9.22)$ & $+106( \pm 135)$ & 0.035 & $7.83( \pm 3.35)$ & $-24( \pm 32)$ & 0.059 \\
\hline Ciprofloxacin $*$ & $0.03( \pm 0.01)$ & $0.11( \pm 0.09)$ & $+316( \pm 363)$ & 0.034 & $0.12( \pm 0.10)$ & $-1( \pm 20)$ & 1.0 \\
\hline Trimethoprim/sulphamethoxazole * & $0.09( \pm 0.02)$ & $0.14( \pm 0.06)$ & $+58( \pm 55)$ & 0.014 & $0.12( \pm 0.05)$ & $-7( \pm 17)$ & 0.371 \\
\hline Tetracycline $*$ & $2.42( \pm 0.90)$ & $23.29( \pm 25.98)$ & $+776( \pm 1027)$ & 0.001 & $21.92( \pm 25.02)$ & $-1( \pm 22)$ & 0.423 \\
\hline Ampicillin ** & $18.6( \pm 1.5)$ & $12.0( \pm 6.4)$ & $+35( \pm 35)$ & 0.014 & $11.8( \pm 6.2)$ & $-1( \pm 3)$ & 1.0 \\
\hline
\end{tabular}

${ }^{*} \mathrm{MIC}(\mu \mathrm{g} / \mathrm{mL})$; ${ }^{* *}$ Diameter of zone inhibition $(\mathrm{mm})$; SD: Standard Deviation. 

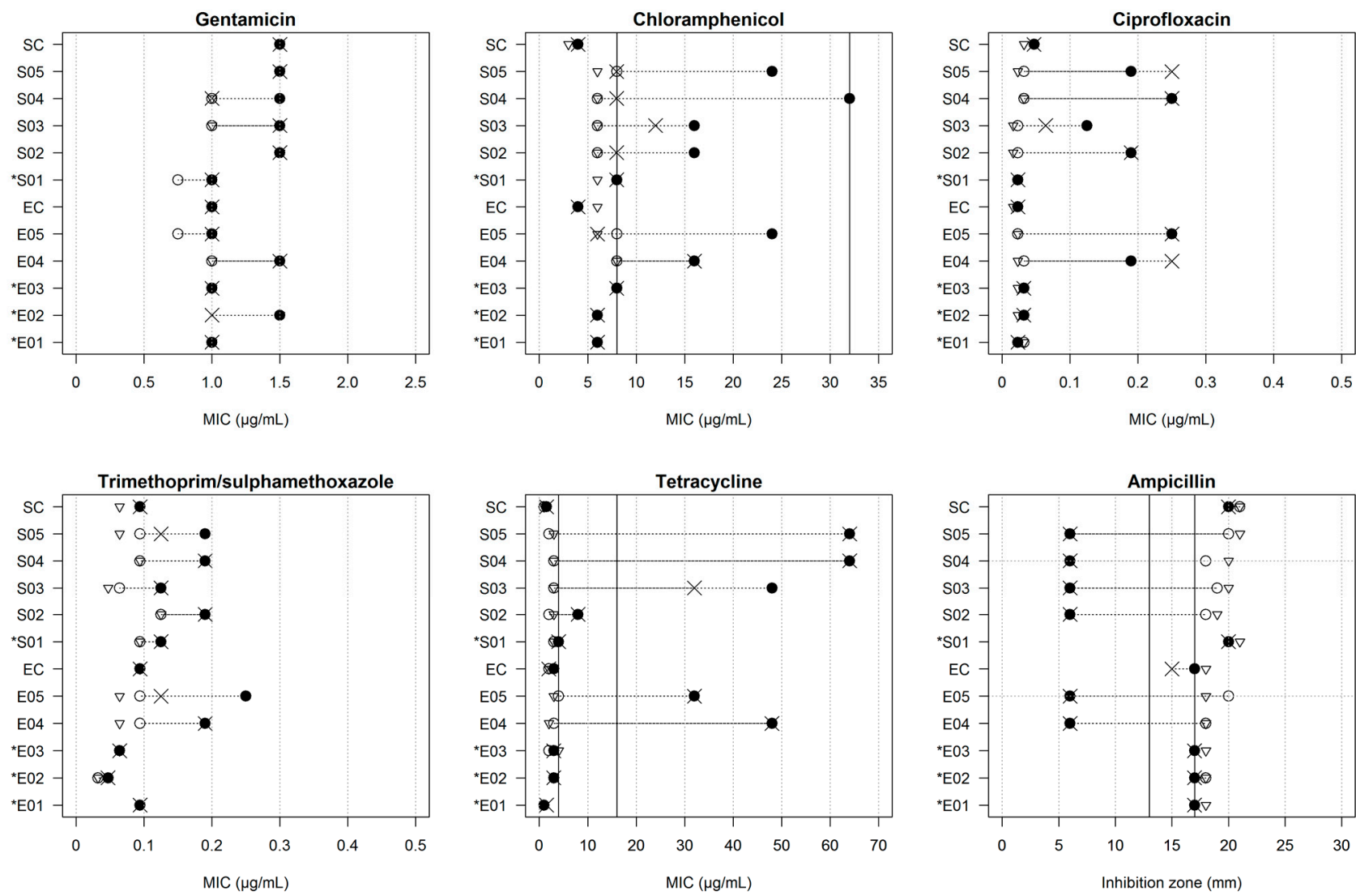

Figure 2. MIC $(\mu \mathrm{g} / \mathrm{mL})$ for antimicrobials of 12 strains before $(\circ)$ and after $(\bullet)$ exposure experiment; controls of adaptation experiment $(\nabla)$ and after treatment with efflux pump inhibitor PA $\beta N(x)$. Vertical black lines: breakpoints for susceptible (on the left), intermediate (between two lines), and resistant (on the right) classes based on Clinical and Laboratory Standard Institute (CLSI) guidelines. For ampicillin, changes in inhibition zone diameter $(\mathrm{mm})$ shown, with full resistance on the left and susceptibility on the right. Breakpoints for gentamicin ( $\leq 4 \mu \mathrm{g} / \mathrm{mL}$, sensitive), ciprofloxacin $(\leq 1 \mu \mathrm{g} / \mathrm{mL}$, sensitive), and trimethoprim/sulphamethoxazole $(\leq 2 \mu \mathrm{g} / \mathrm{mL}$, sensitive) not shown since outside the range. * Strains did not increase MIC for Product A.

The changes in MIC or the zone diameter of strains cultured in disinfectant-free media (compared to pre-exposure strains) were $-19.9 \%(\mathrm{SD} \pm 14.6),-4.2 \%(\mathrm{SD} \pm 20.9),-12.8 \%(\mathrm{SD} \pm 15.9),+5.5 \%$ $(\mathrm{SD} \pm 13.0),+11.8 \%(\mathrm{SD} \pm 37.0)$, and $+3.3 \%(\mathrm{SD} \pm 5.3)$ for ciprofloxacin, chloramphenicol, trimethoprim/sulphamethoxazole, gentamicin, tetracycline, and ampicillin, respectively. None of these changes were statistically significant ( $p>0.05$, Wilcoxon tests).

\subsection{Impact of Efflux Pump Blocking on MIC of Product A and Antimicrobial Resistance}

All strains were treated with the generic efflux pump inhibitor phenyl-arginine beta-naphthylamide $(\mathrm{PA} \beta \mathrm{N})$ after the adaptation experiment. This resulted in an overall mean reduction of $18 \%(\mathrm{SD} \pm 15)$ in the MIC for Product A (from $27 \mu \mathrm{g} / \mathrm{mL}(\mathrm{SD} \pm 12$ )) after exposure to Product A to $21 \mu \mathrm{g} / \mathrm{mL}$ (SD \pm 9 ) after treatment with $\mathrm{PA} \beta \mathrm{N})(p=0.04$; paired Wilcoxon test) (Table 1$)$. The observed variability in 
observed MIC changes between experimental replicates was moderate, with a median coefficient of variation of $17.3 \%$ (IQR $0-45 \%$ ).

Treatment with PA $\beta \mathrm{N}$ did not result in significant changes in the susceptibility of antimicrobials with the exception of chloramphenicol (mean reduction of $24 \%(\mathrm{SD} \pm 35), p=0.059)$. A total of four strains (three NTS and one E. coli) reverted to being fully susceptible to chloramphenicol after treatment with PAßN (Table 2 and Figure 2).

\subsection{Impact of Efflux Pump Blocking on AMR among Representative E. coli Isolates from Animals}

After treatment with $\mathrm{PA} \beta \mathrm{N}, E$. coli isolates increased their inhibition zone diameters in the disc diffusion test to all investigated antimicrobials ( $p \leq 0.002$, paired Wilcoxon test). The percent of strains that increased their zone diameter by at least $1 \mathrm{~mm}$ was greatest for ciprofloxacin $(39.3 \%)$, followed by chloramphenicol (27.1\%), gentamicin (25.2\%), ampicillin (16.8\%), trimethoprim/sulphamethoxazole (16.8\%), and tetracycline (7.7\%) (Figure $\mathrm{S} 1)$.

However, the categorical changes in the observed prevalence of AMR (intermediate and fully resistant taken together) based on CLSI guidelines before and after treatment with PA $\beta \mathrm{N}$ were much more modest. The treatment resulted in reductions in absolute levels of resistance prevalence of $3.3 \%$, $2.6 \%, 2.6 \%$, and $0.7 \%$ for chloramphenicol, ciprofloxacin, ampicillin, and gentamicin, respectively. A total of $7.8 \%$ isolates changed their profile from fully resistant to intermediate resistant for ampicillin. No changes in the prevalence of resistance for trimethoprim/sulphamethoxazole and tetracycline were observed (Table 3).

Table 3. Prevalence (\%) of antimicrobial resistance among 155 representative E. coli isolates from animals before and after treatment with efflux pump inhibitor (PA $\beta \mathrm{N})$.

\begin{tabular}{|c|c|c|c|c|c|c|c|c|c|}
\hline \multirow{2}{*}{ Antimicrobial } & \multicolumn{4}{|c|}{ Before Treatment with PABN } & \multicolumn{4}{|c|}{ After Treatment with PAßN } & \multirow{2}{*}{$\begin{array}{l}\text { Relative } \\
\text { Change }\end{array}$} \\
\hline & $\mathbf{S}$ & $\mathbf{I}$ & $\mathbf{R}$ & $\mathbf{I}+\mathbf{R}$ & $\mathbf{S}$ & $\mathbf{I}$ & $\mathbf{R}$ & $\mathbf{I}+\mathbf{R}$ & \\
\hline Gentamicin & 80.0 & 5.2 & 14.8 & 20.0 & 80.7 & 4.5 & 14.8 & 19.3 & -0.7 \\
\hline Chloramphenicol & 43.8 & 5.2 & 51.0 & 56.2 & 47.1 & 1.9 & 51.0 & 52.9 & -3.3 \\
\hline Ciprofloxacin & 70.3 & 12.9 & 16.8 & 29.7 & 72.9 & 10.3 & 16.8 & 27.1 & -2.6 \\
\hline Trimethoprim/sulphamethoxazole & 40.0 & 1.3 & 58.7 & 60.0 & 40.0 & 1.3 & 58.7 & 60.0 & 0 \\
\hline Tetracycline & 23.9 & 0.1 & 76.0 & 76.1 & 23.9 & 0.1 & 76.0 & 76.1 & 0 \\
\hline Ampicillin & 12.9 & 16.1 & 71.0 & 87.1 & 15.5 & 21.3 & 63.2 & 84.5 & -2.6 \\
\hline
\end{tabular}

S: Fully sensitive; I: Intermediate resistant; R: Fully resistant.

\section{Discussion}

This is one of few studies demonstrating an association between increases in MIC for a commercial QAC- and glutaraldehyde-based disinfectant and AMR on field Enterobacteriacea (E. coli and NTS) strains. Although the investigated product is widely used on food animal farms in southern Vietnam, the chosen strains originated from healthy human individuals since we expected such isolates have a low chance of previous exposure to disinfectants commonly used on pig and poultry farms.

After a 12-day adaptation period, increases in MIC for a commercial disinfectant product containing a QAC and glutaraldehyde ranged from $0 \%$ to $+100 \%$ (median $+31 \%$ ). Previous adaptation experiments using E. coli referent strains gradually exposed to benzalkonium chloride resulted in 
comparatively greater MIC changes ranging from $+500 \%-600 \%$ [10,17]. Another study on a Salmonella Virchow isolate resulted in an even greater MIC increase for benzalkonium chloride from $4 \mu \mathrm{g} / \mathrm{mL}$ to $256 \mu \mathrm{g} / \mathrm{mL}$, i.e., a $+6300 \%$ increase [18]. Such experiments were carried out using pure benzalkonium chloride; in contrast, in our study we investigated a commercial product that contains a mixture of benzalkonium chloride and glutaraldehyde, and therefore adaptation to either of the two components could theoretically result in an increased MIC for the commercial product. In addition to benzalkonium chloride, the phenomenon of adaptation has also been described with other QACs commonly used in the food industry and animal production such as dimethyl didecyl ammonium chloride (DDAC) and dimethyl dioctyl ammonium chloride (OCDAC) [19].

The observed increases in MIC for the disinfectant product were strongly associated with marked increases in MIC (or decreases in inhibition zone) for all antimicrobials tested. The greatest changes were observed in decreasing order for ampicillin, tetracycline, ciprofloxacin, chloramphenicol, trimethoprim/sulphamethoxazole, and gentamicin. These results are consistent with previous studies investigating cross-resistance between QAC-based disinfectants and a range of antimicrobials [10,1720]. Interestingly, our results suggest that the tested product did not contribute to changes in the observed resistance pattern for the aminoglycoside (i.e., gentamicin), which is in agreement with a previous study investigating cross-resistance between QACs and a range of antimicrobials [19]. The fact that an association between a commercial disinfectant and ciprofloxacin was shown is of particular concern since fluoroquinolones are defined as antimicrobials of critical importance for human medicine [21].

Recent studies by our research group on livestock and poultry farms in southern Vietnam showed that ampicillin and tetracycline were the two antimicrobials (of eight tested) against which E. coli strains exhibited the highest levels of resistance [14]. Similarly, these two antimicrobials were the first and third (of 10 tested) against which NTS isolates showed the highest resistance levels [15]. Furthermore, E. coli from small wild mammals (rats and shrews) trapped on the same farms consistently showed the highest levels of AMR against those two antimicrobials. Since wild mammals are not deliberately treated with antimicrobials, we hypothesize that this may partly be a reflection of co-resistance due to the high volume of disinfectants or antimicrobials that may be discharged into the environment. In contrast to glutaraldehyde, which is readily biodegradable in soil and freshwater [22], QACs are generally considered to have poor biodegradability and are mainly excreted in nonmetabolized form [23].

A limitation of our study is the relatively small number of strains investigated. This was dictated by laboratory capacity and the high costs associated with MIC testing for a large number of antimicrobials. In spite of this, the direction of the changes observed (a clear increase in MIC for Product A after the adaptation experiment) and the observed strong association between these changes and the observed increases in MIC for antimicrobials strongly support the phenomenon of crossresistance. In contrast to small replicate variation, there were considerable differences in MIC results between strains, which could theoretically result in potential biases due the choice of strains.

Treatment of strains with a generic efflux pump inhibitor (PA $\beta N)$ after the adaptation experiment did not result in significant changes in their MIC for antimicrobials with the exception of chloramphenicol (four strains reverted to full susceptibility). Furthermore, the changes observed after treatment of field strains with PA $\beta \mathrm{N}$ were modest, although in the direction of increased susceptibility. 
In the case of ampicillin, a significant fraction (7.8\%) of field isolates changed their category from fully resistant to intermediate resistant after treatment with PA $\mathrm{N}$. This is surprising given the low level of reversion of resistance against this antimicrobial observed in Product A-adapted strains after treatment with $\mathrm{PA} \beta \mathrm{N}$. This suggests that the AMR phenotypic expression of induced cross-resistance between the disinfectant product and antimicrobials may be due to mechanisms other than generic efflux pump expression such as gene mutations conferring inactivation of porins or overexpression of glycolitic enzymes $[10,20]$. Alternatively, given the potential huge diversity of substrate binding sites present in efflux pumps [24] and the lack of information about the specific efflux pumps in our strains, the use of a single universal inhibitor such as PA $\beta N$ may not have been sufficient to completely block the efflux pump activity in the bacterial cell [25].

In the Mekong Delta of Vietnam over $80 \%$ of poultry farms routinely use QAC-based disinfectants, and $74 \%$ of farms surveyed use the commercial product tested here, most of them on occupied buildings (Table S1). This is a concern, since in the presence of animal waste disinfectants may be diluted, causing bacterial populations to be exposed to sub-optimal doses for long periods of time, therefore promoting the rapid development of resistance [26].

\section{Material and Methods}

\subsection{Study Design}

We investigated pan-susceptible Enterobacteriaceae strains of human origin that were gradually exposed to increasing concentrations of a benzalkonium chloride- and glutaraldehyde-containing commercial Product A over a 12-day period. Strains were investigated for their antimicrobial susceptibilities against gentamicin, chloramphenicol, ciprofloxacin, trimethoprim/sulphamethoxazole, tetracycline, and ampicillin before and after the adaptation period. The potential induction of efflux pumps in disinfectant/antimicrobial cross-resistance mechanisms was investigated by incubating bacterial strains after the adaptation experiments with $\mathrm{PA} \beta \mathrm{N}$, a generic efflux pump inhibitor and subsequent AMR testing. We also treated with PA $\beta \mathrm{N}$ a sample of representative E. coli isolates of farm animal origin to investigate the overall potential contribution of efflux pumps on observed levels of AMR.

\subsection{Selection of Strains and AMR Testing}

Six E. coli and six NTS strains were used. Of those, 10 isolates (five E. coli and five NTS strains) were obtained from healthy human subjects as part of a larger study investigating AMR in humans and animals in Tien Giang Province (Vietnam). In addition, two fully susceptible laboratory strains, Salmonella Typhimurium 14028 and E. coli 25922, were included for quality control of susceptibility tests. E. coli isolates were confirmed using standard biochemical tests (motility, indole, lactose/glucose fermentation, methyl red, citrate, urease, hydrogen sulfide, and gas production). NTS were isolated using selective media (Rambach and Xylose Lysine Deoxycholate agar) and confirmed by serological method (PSO and PSH polyvalent). All selected strains were sensitive against the six antimicrobials investigated using the Bauer-Kirby disc diffusion method according to Clinical and Laboratory Standard Institute (CLSI) procedures [27]: gentamicin (CN, $10 \mu \mathrm{g})$, chloramphenicol (C, $30 \mu \mathrm{g})$, 
ciprofloxacin (CIP, $5 \mu \mathrm{g}$ ), trimethoprim/sulphamethoxazole (SXT, 1.25/23.75 $\mu \mathrm{g}$ ), tetracycline (TE, $30 \mu \mathrm{g}$ ), ampicillin (AMP, $10 \mu \mathrm{g}$ ) (Oxoid, Hampshire, UK). Etest strips (BioMerieux, Marcy I'Etoile, France) were used to determine the minimum inhibitory concentration (MIC) for gentamicin (GM256), chloramphenicol (CL256), ciprofloxacin (CI32), trimethoprim/sulphamethoxazole (TS32), and tetracycline (TC256). Since the Etest of ampicillin was not available in our laboratory, AMR for ampicillin was tested using disc diffusion method (AMP, $10 \mu \mathrm{g}$ ).

\subsection{Adaptation Experiment to Product A}

Product A, a commercial disinfectant containing benzalkonium chloride $(150 \mathrm{mg} / \mathrm{mL})$ and glutaraldehyde $(150 \mathrm{mg} / \mathrm{mL})$ as main active ingredients, was used in the adaptation experiment.

The baseline MIC for Product A corresponded to the lowest concentration of the product that prevents bacterial growth after incubation for $24 \mathrm{~h}$ at $37{ }^{\circ} \mathrm{C}$. The obtained MIC represents the concentration of active components (both benzalkonium and glutaraldehyde). The MIC was first determined for each strain using a serial dilution method: from stock solution of Product A, serial dilutions ranging from 60 to $300 \mu \mathrm{g} / \mathrm{mL}$ in distilled water were prepared. $200 \mu \mathrm{L}$ of each dilution was added to $1.8 \mathrm{~mL}$ of $\mathrm{MH}$ broth (Oxoid, Hampshire, UK) containing a bacterial suspension with $10^{5}$ $\mathrm{CFU} / \mathrm{mL}$ and incubated for $24 \mathrm{~h}$ at $37^{\circ} \mathrm{C}$. Bacterial growth was assessed by plating into $\mathrm{MH}$ agar (Oxoid, Hampshire, UK), followed by incubation for $24 \mathrm{~h}$.

The adaptation experiment was performed according to a previously described procedure [19]. Briefly, colonies were suspended in $\mathrm{MH}$ broth and incubated for $24 \mathrm{~h}$ at $37^{\circ} \mathrm{C}$. An aliquot of $50 \mu \mathrm{L}$ of a calibrated suspension containing $10^{8} \mathrm{CFU} / \mathrm{mL}$ of each strain was initially mixed with an initial concentration of $0.5 \mathrm{MIC}$ of Product $\mathrm{A}$ in a total volume of $5 \mathrm{~mL}$ of $\mathrm{MH}$ broth, and incubated for $24 \mathrm{~h}$ at $37{ }^{\circ} \mathrm{C}$. After incubating, cultures were calibrated to concentration of $10^{8} \mathrm{CFU} / \mathrm{mL}$. Then $50 \mu \mathrm{L}$ of suspension was transferred to $5 \mathrm{~mL}$ of $\mathrm{MH}$ broth containing higher concentration of disinfectant. The cultures were also plated onto $\mathrm{MH}$ agar for growth confirmation and storage. The procedure was repeated daily over 12 days or stopped earlier if no growth was observed. The highest concentration of Product A in which bacteria could grow was used. The observed change in MIC before and after the adaptation experiment was recorded in absolute terms $(\mu \mathrm{g} / \mathrm{mL})$ for each experimental replicate. The reported MIC to Product A before and after adaptation was calculated as the average of the three experimental replicates. In order to exclude the potential development of MIC changes due to spontaneous mutation as a result of exposure to disinfectant-free nutrient broth, all 12 strains were tested for their MIC of Product A after a 12-day period in $\mathrm{MH}$ broth at $37{ }^{\circ} \mathrm{C}$. All strains were investigated in triplicate.

\subsection{Investigation of the Changes in AMR before and after Exposure to Product A}

For all 12 E. coli and NTS strains the MIC changes (gentamicin, chloramphenicol, trimethoprim/ sulphamethoxazole, ciprofloxacin, tetracycline) as well as zone diameter change (ampicillin) were investigated before and after the adaptation experiment. In addition, potential changes in the AMR profile (MIC and zone diameter) due to exposure to disinfectant-free nutrient broth was investigated among all studied strains after a 12-day period in $\mathrm{MH}$ broth at $37^{\circ} \mathrm{C}$. 


\subsection{Investigation of the Role of Efflux Pumps on Antimicrobial Resistance}

The potential contribution of efflux pumps on the observed pattern of resistance was investigated by treating all 12 studied strains with $50 \mu \mathrm{g} / \mathrm{mL}$ of the generic efflux pump inhibitor phenyl-arginine beta-naphthylamide (PA $\beta N$ ) (Sigma, Munich, Germany) [25] as described elsewhere [28]. The observed changes in MIC before and after treatment with PA $\beta \mathrm{N}$ for each experimental replicate were recorded in absolute terms $(\mu \mathrm{g} / \mathrm{mL})$. The reported MIC to Product A before and after adaptation was calculated as the average of the three experimental replicates. In addition, a selection of 155 representative $E$. coli isolates from chicken, duck, pig farms and small wild mammals trapped on farms and rice field/forest sites from the Mekong Delta region of Vietnam [14] were tested for AMR (disc diffusion method) before and after treatment with $50 \mu \mathrm{g} / \mathrm{mL}$ of PA $\beta \mathrm{N}$.

\subsection{Statistical Analyses}

Changes in the MIC for Product A before and after the adaptation experiment and after treatment with $\mathrm{PA} \beta \mathrm{N}$ were compared using the non-parametric paired Wilcoxon signed-rank test. The potential statistical association between observed changes in MIC for Product A and AMR as a result of the adaptation experiments was investigated using Pearson's correlation test. Kruskal-Wallis paired tests were used to compare MIC for Product A between E. coli and NTS before and after the adaptation experiment. Analyses were carried out using the "epicalc" package in R software (http://www.rproject.org/).

\section{Conclusions}

We conclusively demonstrated in vitro the phenomena of cross-resistance between benzalkonium chloride- and glutaraldehyde-based disinfectant and antimicrobials. The data suggests that only a small fraction of the observed AMR may be due to generic efflux pumps and more research is required to determine the molecular mechanisms responsible for these changes. Given the widespread and often inadequate use of disinfectants on farms in Vietnam, we suggest that this could potentially be a factor contributing to the observed high levels of AMR among Enterobacteriaceae. We recommend performing well-planned longitudinal studies to investigate molecular changes in organisms before and after exposure to such disinfectants. We also strongly recommend better training on the correct use of disinfectants on farms, since preserving the efficacy of disinfectants is crucial to maintaining hygiene levels on farms and reducing the need for using antimicrobials.

\section{Acknowledgments}

We would like to thank Thai Quoc Hieu, Ho Huynh Mai, Vo Be Hien, Bach Tuan Kiet and the staff of the Sub-Department of Animal Health of Tien Giang and Dong Thap provinces, Vietnam, for their support in field data and sample collection. We are also indebted to Dr. Constance Schultsz (AMC, Amsterdam, The Netherlands) for support during this study. This work has been co-funded by the Vietnam Initiative on Zoonotic Infections (VIZIONS WT/093724), the part of the Wellcome Trust Major Overseas Programme (UK), by the VIBRE Project (ZoNMW/WOTRO/205100012, The Netherlands) and by the Li-Ka-Shing Foundation (83GR). 


\section{Author Contributions}

Nguyen T. Nhung and Juan Carrique-Mas conceived the idea for the project. Stephen Baker, Guy Thwaites, Ngo T. Hoa, James Campbell and Nguyen V. Trung advised on the study design. Nguyen T. Nhung and Cao T. Thuy carried out the laboratory experiments. Juan Carrique-Mas and Nguyen T. Nhung conducted data analyses and produced first draft of paper. All authors commented on subsequent versions.

\section{Conflicts of Interest}

The authors declare no conflict of interest.

\section{References}

1. WHO. Antimicrobial Resistance: Global Report on Surveilance. Available online: http://www.who.int/iris/bitstream/10665/112642/1/9789241564748_eng.pdf (accessed on 30 June 2014).

2. Vallat, B. Antimicrobial resistance in animal and public health. Preface. Rev. Sci. Tech. 2012, 31, 9-14.

3. Chantziaras, I.; Boyen, F.; Callens, B.; Dewulf, J. Correlation between veterinary antimicrobial use and antimicrobial resistance in food-producing animals: A report on seven countries. $J$. Antimicrob. Chemother. 2014, 69, 827-834.

4. Bronzwaer, S.L.; Cars, O.; Buchholz, U.; Molstad, S.; Goettsch, W.; Veldhuijzen, I.K.; Kool, J.L.; Sprenger, M.J.; Degener, J.E. A european study on the relationship between antimicrobial use and antimicrobial resistance. Emerg. Infect. Dis. 2002, 8, 278-282.

5. Fraise, A.P.; Jean-Yves, M.; Sattar, S. Russell, Hugo and Ayliffe's Principles and Practice of Disinfection, Preservation and Sterilization; Wiley-Blackwell: Hoboken, NJ, USA, 2013.

6. Kahrs, R.F. General disinfection guidelines. Rev. Sci. Tech. 1995, 14, 105-163.

7. Davin-Regli, A.; Pages, J.M. Cross-resistance between biocides and antimicrobials: An emerging question. Rev. Sci. Tech. 2012, 31, 89-104.

8. Walsh, S.E.; Maillard, J.Y.; Russell, A.D.; Catrenich, C.E.; Charbonneau, D.L.; Bartolo, R.G. Development of bacterial resistance to several biocides and effects on antibiotic susceptibility. J. Hosp. Infect. 2003, 55, 98-107.

9. Meyer, B.; Cookson, B. Does microbial resistance or adaptation to biocides create a hazard in infection prevention and control? J. Hosp. Infect. 2010, 76, 200-205.

10. Bore, E.; Hebraud, M.; Chafsey, I.; Chambon, C.; Skjaeret, C.; Moen, B.; Moretro, T.; Langsrud, O.; Rudi, K.; Langsrud, S. Adapted tolerance to benzalkonium chloride in Escherichia coli k-12 studied by transcriptome and proteome analyses. Microbiology 2007, 153, 935-946.

11. Buffet-Bataillon, S.; Tattevin, P.; Bonnaure-Mallet, M.; Jolivet-Gougeon, A. Emergence of resistance to antibacterial agents: The role of quaternary ammonium compounds-A critical review. Int. J. Antimicrob. Agents 2012, 39, 381-389. 
12. Hegstad, K.; Langsrud, S.; Lunestad, B.T.; Scheie, A.A.; Sunde, M.; Yazdankhah, S.P. Does the wide use of quaternary ammonium compounds enhance the selection and spread of antimicrobial resistance and thus threaten our health? Microb. Drug Resist. 2010, 16, 91-104.

13. Blair, J.M.; Piddock, L.J. Structure, function and inhibition of rnd efflux pumps in gram-negative bacteria: An update. Curr. Opin. Microbiol. 2009, 12, 512-519.

14. Nhung, N.T.; Cuong, N.V.; Campbell, J.; Hoa, N.T.; Bryant, J.E.; Truc, V.N.; Kiet, B.T.; Jombart, T.; Trung, N.V.; Hien, V.B.; et al. High levels of antimicrobial resistance among Escherichia coli isolates from livestock farms and synanthropic rats and shrews in the Mekong Delta of Vietnam. Appl. Environ. Microbiol. 2015, 81, 812-820.

15. Tu, L.T.; Hoang, N.V.; Cuong, N.V.; Campbell, J.; Bryant, J.E.; Hoa, N.T.; Kiet, B.T.; Thompson, C.; Duy, D.T.; Phat, V.V.; et al. High levels of contamination and antimicrobial-resistant nontyphoidal salmonella serovars on pig and poultry farms in the Mekong Delta of Vietnam. Epidemiol. Infect. 2015, 143, 3074-3086.

16. Trung, N.V.; Carrique-Mas, J.J.; Hoa, N.T.; Mai, H.H.; Tuyen, H.T.; Campbell, J.I.; Nhung, N.T.; Nhung, H.N.; van Minh, P.; Wagenaar, J.A.; et al. Prevalence and risk factors for carriage of antimicrobial-resistant Escherichia coli on household and small-scale chicken farms in the Mekong Delta of Vietnam. J. Antimicrob. Chemother. 2015, doi:10.1093/jac/dkv053.

17. Langsrud, S.; Sundheim, G.; Holck, A.L. Cross-resistance to antibiotics of Escherichia coli adapted to benzalkonium chloride or exposed to stress-inducers. J. Appl. Microbiol. 2004, 96, 201-208.

18. Braoudaki, M.; Hilton, A.C. Adaptive resistance to biocides in Salmonella enterica and Escherichia coli 0157 and cross-resistance to antimicrobial agents. J. Clin. Microbiol. 2004, 42, 73-78.

19. Soumet, C.; Fourreau, E.; Legrandois, P.; Maris, P. Resistance to phenicol compounds following adaptation to quaternary ammonium compounds in Escherichia coli. Vet. Microbiol. 2012, 158, 147-152.

20. Karatzas, K.A.; Randall, L.P.; Webber, M.; Piddock, L.J.; Humphrey, T.J.; Woodward, M.J.; Coldham, N.G. Phenotypic and proteomic characterization of multiply antibiotic-resistant variants of Salmonella enterica serovar typhimurium selected following exposure to disinfectants. Appl. Environ. Microbiol. 2008, 74, 1508-1516.

21. WHO. Critically Important Antimicrobials for Human Medicine; Available online: http://www.who.int/iris/bitstream/10665/77376/1/9789241504485_eng.pdf (accessed on 30 October 2015).

22. Leung, H.W. Ecotoxicology of glutaraldehyde: Review of environmental fate and effects studies. Ecotoxicol. Environ. Saf. 2001, 49, 26-39.

23. Thorsteinsson, T.; Loftsson, T.; Masson, M. Soft antibacterial agents. Curr. Med. Chem. 2003, 10, 1129-1136.

24. Putman, M.; Koole, L.A.; van Veen, H.W.; Konings, W.N. The secondary multidrug transporter lmrp contains multiple drug interaction sites. Biochemistry 1999, 38, 13900-13905.

25. Lomovskaya, O.; Warren, M.S.; Lee, A.; Galazzo, J.; Fronko, R.; Lee, M.; Blais, J.; Cho, D.; Chamberland, S.; Renau, T.; et al. Identification and characterization of inhibitors of multidrug 
resistance efflux pumps in Pseudomonas aeruginosa: Novel agents for combination therapy. Antimicrob. Agents Chemother. 2001, 45, 105-116.

26. Fraise, A.P. Biocide abuse and antimicrobial resistance-A cause for concern? J. Antimicrob. Chemother. 2002, 49, 11-12.

27. CLSI. Performance Standards for Antimicrobial Susceptibility Testing; Twenty-First Informational Supplement; Clinical and Laboratory Standards Institute: Wayne, PA, USA, 2011; Volume CLSI/NCCLS M100-S21.

28. Lamers, R.P.; Cavallari, J.F.; Burrows, L.L. The efflux inhibitor phenylalanine-arginine beta-naphthylamide (pabetan) permeabilizes the outer membrane of gram-negative bacteria. PLOS ONE 2013, 8, e60666.

(C) 2015 by the authors; licensee MDPI, Basel, Switzerland. This article is an open access article distributed under the terms and conditions of the Creative Commons Attribution license (http://creativecommons.org/licenses/by/4.0/). 